\title{
COVID-19: Exploring the Disease Transmission Dynamics
}

\section{Nikhra $V^{*}$}

Department of Medicine, Hindu Rao Hospital \& NDMC Medical College, India

*Corresponding author: Vinod Nikhra, M.D, Senior Consultant and Faculty, Department of Medicine, Hindu Rao Hospital and NDMC Medical College, New Delhi, India, Email: drvinodnikhra@gmail.com

\section{Mini Review \\ Volume 2 Issue 2}

Received Date: November 18, 2020

Published Date: December 08, 2020

DOI: $10.23880 /$ aii-16000129

\section{Abstract}

The Immuno-Thrombo-Inflammatory Disease: COVID-19 is an acute immuno-thrombogenic inflammatory viral disease manifested by dysfunctions related to multiple organs involved in its pathogenesis. Its incidence and prevalence is related to environmental viability of the virus, various transmission factors associated with the agent and the host, possible modes of transmission, period of infectiousness, and composition and susceptibility of the population. Whereas respiratory route is dominant mode of transmission, transmission through direct contact and fomite transmission do occur.

SARS-CoV-2: Understanding the Agent Factors: Understanding of SARS-CoV-2 or hCoV-19 structural components is important for understanding the dynamics of the disease transmission and propagation. The virus has a lipid shell, a singlestranded RNA genome containing 29891 nucleotides, spike, envelope, and membrane and hemagglutinin-esterase dimer proteins. The SARS-CoV-2 structural components have been related to the COVID-19 pathogenic mechanisms.

The Kinetics of Transmission and Propagation: Transmission of SARS-CoV- 2 to cause COVID-19 requires that a minimum but unknown dose of replication-competent virus be delivered to an appropriate anatomical site in a susceptible and vulnerable host. A combination of various agent (viral), host, and environmental factors influence the transmission and course of the disease.

Fear, Confusion, and Impact on Mass Behavior: The un-relented spread of COVID-19 pandemic is a major public health concern threatening general and mental health and safety of the human life all over the globe. The associated anxiety and emotional stress levels are often high. The healthcare professionals, too, suffer with various concerns like long and strenuous working hours, being able to provide competent medical care, their safety at the workplace and taking the infection home to their family, and uncertainty about their organizational support for their personal and family needs.

Conclusion: The Lessons for Pandemic Control: The human subjects produce respiratory droplets ranging from 0.1 to $1000 \mu \mathrm{m}$. Depending on droplet size, inertia, gravity, and evaporation factors, the emitted droplets and aerosols disperse in air. There are two obvious transmission pathways: the airborne inhalation and contracting through the contaminated surfaces. It appears that the contaminated surfaces may play less significant role as compared to the infected airborne droplets and the aerosol in the disease transmission.

Keywords: COVID-19; COVID-19 Strains; D614G Variant; Gly614 Variant; SARS-CoV-2; Spike Protein; Transmission Kinetics; Virus Mutations 
Abbreviations: S: Spike protein; N: Nucleocapsid Protein; RTC: Replicase-Transcriptase Complex; ACE2: AngiotensinConverting Enzyme II; E: Envelope; M: Membrane Protein; HE: Hemagglutinin-Esterase; PRRs: Pattern Recognition Receptors; IFN-g: Interferon Gamma, TNFs: Tumor Necrosis Factors, ILs: Interleukins; JNK: c-Jun N-Terminal Kinase; DIC: Disseminated Intravascular Coagulation; MOF: Multi-Organ Failure; RT: Replication-Transcription; ORFs: Open Reading Frames; nsps: Non-Structural Proteins; RBD: Receptor Binding Domain; G: Glycine; PPE: Personal Protective Equipment.

\section{The Immuno-Thrombo-Inflammatory Viral Disease}

\section{The Virus and the Disease}

The COVID-19 is an acute immuno-thrombogenic inflammatory viral disease manifested by dysfunctions related to multiple organs involved in its pathogenesis. Further, the incidence and prevalence of the disease have been related to the environmental viability of the virus, various transmission factors associated with the agent and the host, possible modes of transmission, period of infectiousness, and composition and susceptibility of the population [1]. Whereas the respiratory route is common and the dominant mode of transmission, transmission through direct contact and fomite transmission do occur. Other modes, such as through sexual, fecal-oral, and blood borne routes have not been documented. Similarly, there are no confirmed cases suggesting in-utero transmission or through breast-feeding or through domestic pets.

For the transmission through respiratory route, physical proximity is the key determinant with the densely packed and ill-ventilated spaces accentuating the probability of transmission. In the clinical setting, the transmissibility correlates with severity of symptoms, albeit the asymptomatic patients also transmit the infection complicating the disease epidemiology. The period of infectiousness has been documented to be about 9 days of symptomatic phase, though the detectable virus shedding may persist for longer periods [2]. In addition, it is also associated with the duration and frequency of exposure and linked to the contracted viral load.

\section{COVID-19 Scenario in Developing Countries}

Considering in a totality, the COVID-19 cases in developing countries are more than the developed countries. The high population density, smaller dwelling units, and lack of quality healthcare, compounded with illiteracy and indifferent attitude of the people and government organisations have been related to a high-incidence setting accompanied with high morbidity and mortality. In the low-income countries, the reported cases and deaths have been concentrated in younger cohorts than expected from observations in higher-income countries, even after accounting for demographic differences across settings. It has been noted that both cases and deaths due to the viral illness have been mostly concentrated in the 40-69 years age group in India as compared to what seen in high-income countries, among other trends [3].

Our current understanding of COVID-19 is largely derived from clinical and epidemiologic studies of the pandemic undertaken in in China and various European countries and North America. There are over 1.3 billion people are at risk of SARS-CoV-2 infection in India. Further challenges exist for the healthcare providers in smaller cities and rural areas, as the COVID-19 pandemic has put a tremendous impact on primary care practices. The population groups and people often fail to comprehend that the pandemic is real. They are uncomfortable with the required prerequisites for social interaction, prevailing uncertainty about their health, economic issues, and various other reasons.

\section{SARS-CoV-2: Understanding the Agent Factors}

Understanding of SARS-CoV-2 or hCoV-19 structural Components is important for understanding the dynamics of the disease transmission and propagation. The virus has a round, elliptical or pleomorphic shape with a diameter of $60-140 \mathrm{~nm}$. It has a single-stranded RNA genome containing 29891 nucleotides, encoding for 9860 amino acids and bearing a sequence identity approximately $50 \%$ to MERS$\mathrm{CoV}$ and $79 \%$ to SARS-CoV. Like other CoVs, it is sensitive to ultraviolet rays and heat and inactivated by lipid solvents including ether and ethanol.

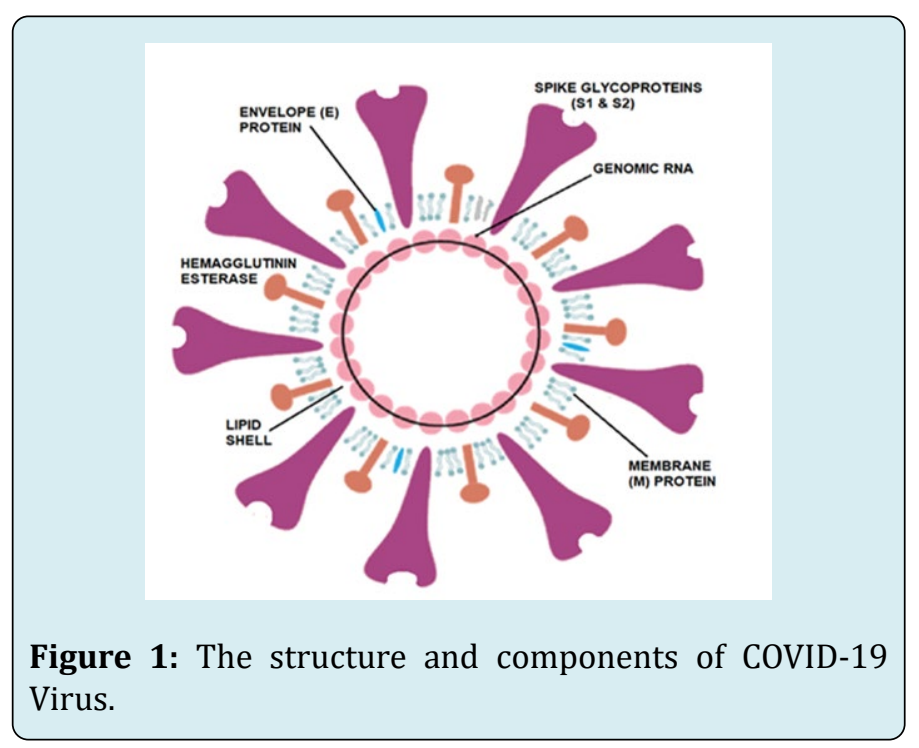


The SARS-CoV-2 virus has a lipid shell, an RNA genome, spike, and envelope and membrane and hemagglutininesterase dimer proteins (Figure 1). The SARS-CoV-2 structural components have been related to the COVID-19 pathogenic mechanisms.

- The glycosylated Spike protein (S) forms the distinct spikes on the surface of the virus. It utilizes an N-terminal signal sequence to gain access to the endoplasmic reticulum and mediates the attachment to host angiotensin-converting enzyme II (ACE2) receptors. The $S$ protein is cleaved by a host cell furin-like protease into two separate polypeptides S1 and S2. The D614G mutation is thought to increase the transmissibility of the virus through enhancing the binding of the $S$ protein to ACE2.

- $\quad$ The viral RNA genome is bound by the phosphorylated nucleocapsid protein $(\mathrm{N})$ in a beads-on-a-string conformation. On entering the host cells, the $\mathrm{N}$ protein potentially tethers the viral genome to replicasetranscriptase complex (RTC). It also helps in packaging the encapsulated genome into viral particles.

- The envelope (E) protein is found in small quantities and appears to have been a trans-membrane ion channel activity. The protein facilitates assembly and release of the new virions. It is related to the disease pathogenesis and important for the disease progression.

- The Membrane protein (M) is the most abundant structural component of the virus. It exists as a dimer and enables to maintain membrane curvature on one end and bound to nucleocapsid proteins on the other.

- The Hemagglutinin-esterase (HE) is also a dimer protein and binds to sialic acids on surface glycoproteins. It is responsible for facilitating and enhancing $S$ proteinmediated cell entry and virus spread through the host mucosal cells.

- SARS-CoV-2 uses a methyl transferase to cap its messenger RNAs to prevent them from being recognized by the host immune system and ensure their translation in host cells [4]. Disrupting the formation of the active methyl transferase complex and/or block its catalytic activity appears to be a potential strategy for developing COVID-19 therapeutics.

\section{Dynamics of Transmission and Propagation}

Transmission of SARS-CoV-2 to cause COVID-19 requires that a minimum but unknown dose of replication-competent virus be delivered to an appropriate anatomical site in a susceptible and vulnerable host. A combination of agent (viral), host, and environmental characteristics influence the transmission. Live virus has been isolated for up to 3hours from aerosols and variable period from various surfaces. The virus is able to persist on plastics and stainless-steel surfaces for longer periods, with the half-life being about 6 hours. In the real-world settings, though the live virus is not detectable on most high-touch surfaces. The virus is stable at low temperatures but sensitive to heat. Various disinfectants may denature the virus.

\section{New Insights into COVID-19 Viral Spread}

- Whereas there is a rising trend in the infection rise as people are returning to their work-places, in other places the outbreaks are related to the younger people crowding and partying care-freely and other people trying to resort back to their earlier ways of life. Because most of the new outbreaks involve the cases in younger age groups, fewer of them develop severe form of the disease. But it is only a matter of time before the elderly people is affected leading to higher mortality. Further, reopening the educational facilities across the continents may make matters worse.

- The airborne transmission: The transmissibility of SARS-CoV-2 is high. The infected individuals release aerosols and droplets containing SARS-CoV-2 while talking, coughing, or sneezing. These virus-containing aerosols and droplets can lead to short-range airborne transmission $(\sim 6 \mathrm{ft})$. Much depends on the size of the aerosols $(<10-\mu \mathrm{m}$ diameter) and droplets $(>10 \mu \mathrm{m}$ diameter) which can promote infection through direct inhalation and deposition on surfaces and subsequent hand-to-mouth/nose/eye transfer (Figure 2).

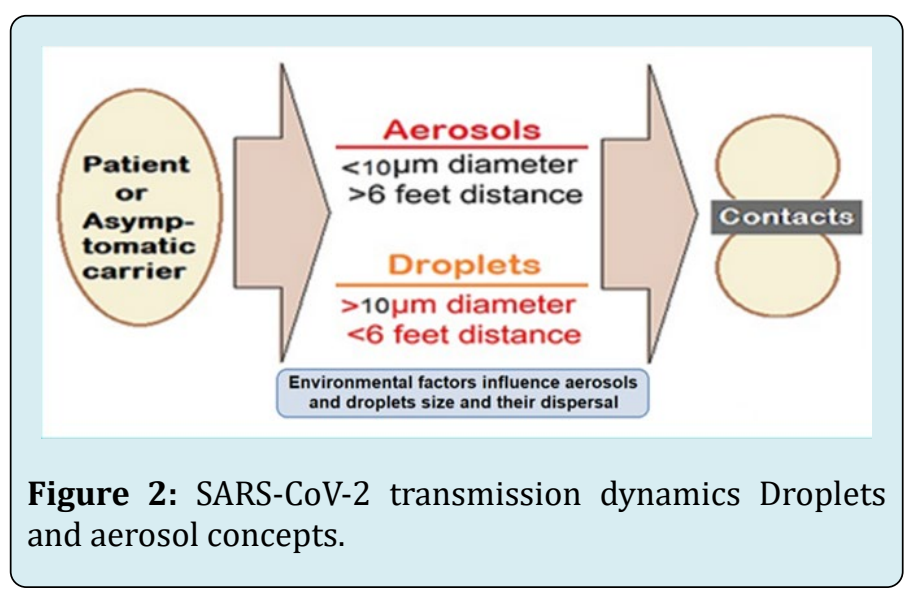

The suspended airborne droplets appear to persist in the air for several minutes. Whereas the smaller aerosols can persist for longer durations (several minutes to hours). The characteristics of aerosols are dynamic, due to evaporative loss of water depending on ventilation, humidity, and temperature levels. With decreasing size, their ability to disperse in air is enhanced, leading to their transmission extending beyond 6 feet from the point of release.

- The aerosol size fractions may influence the deposition profile of SARS-CoV-2 in the lung [5]. Thus, whereas the 
larger aerosols $(>4 \mu \mathrm{m})$ are predominantly deposited in the upper and central airways (i.e., nasopharynx, tracheobronchial) and are subject to muco-ciliary clearance, the smaller aerosols $(<4 \mu \mathrm{m})$ get deposited deeper in the alveoli, having epithelial cells rich in ACE2, with enhanced transmission efficiency.

- The transmission may occur through fomites also but appears to play a minor role (Figure 3). Further, the emphasis on hand hygiene has been diluted, as it is becoming clear that contaminated surfaces may not play a large role. Similarly, the emphasis on banning outdoor activities is losing focus, as it is becoming established that the outdoor activities like jogging, outdoor hospitality, non-essential shopping, and public transportation are fine as long as people keep social distancing and wear face masks. The focus is, rather on indoor activities which are the main culprit for the virus transmission.

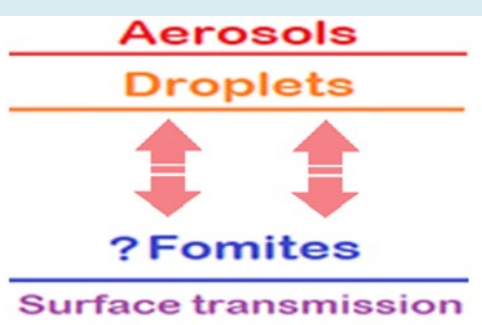

Environmental factors influence the viral viability in air and on surfaces

Figure 3: Transmission kinetics Aerosols, droplets and fomites interlink.

- The emphasis should also be on targeting outbreak clusters and super-spreading events. The studies indicate that 10 percent of patients cause 80 percent of all the infections, whereas most patients to the tune of 90 percent do not infect further. As such, the superspreading events are uncommon [6].

- The backward contact tracing is more useful than the forward tracing. Further, finding clusters help epidemiologists in understanding about the outbreaks. With more stress on the long-term care facilities and workplaces, the specifically targeted measures help in preventing outbreaks, rather than general lockdown measures. These targeted versions include encouraging people to work from home and avoiding crowded places and banning meetings and gatherings.

- Using the preventive measures and resources rationally, various countries are now better equipped than before in the current scenario. We are aware of the disease epidemiology, the virus transmission kinetics, and equipped with resources like PPE kits and masks. Further, the rational behaviour has emerged in place of irrational fears about the disease. In addition, most countries have developed machinery for contact tracing, surveillance and gathering data.

\section{The Viral Transmission and Propagation}

With the infection contracted, the viral load is highest in the upper respiratory tract (nasopharynx and oropharynx) early in disease and then increases in the lower respiratory tract. Susceptibility to SARS-CoV-2 infection increases with age; children $<10$ years (4-7 percent) are around half as susceptible as adults (14-17 percent).

As with other respiratory infections, the transmission occurs through droplets discharged by the infected person or by being carried to oral or nasal mucosa by hands from the virus-infested surfaces. It appears that all COVID-19 patients asymptomatic, mild, or severe shed a massive number of viruses in the surroundings. Aerosol transmission may also be possible in closed and confined spaces. The incubation period is from 3 to 7 days, ad 14 days is being considered as the longest possible time from infection to appearance of symptoms. In addition to the respiratory droplets and direct contact, fecal-oral transmission may also be a route of transmission. It is to be cautioned that the information is derived from the early research and reports and further studies may aid understanding the mechanisms of transmission, the incubation period, duration of infectivity and the clinical course of COVID-19.

\section{Steps in Progression of COVID-19 Illness}

Once inside the airways, the virus through its surface (S) protein tends to recognize and stick to the receptor protein called ACE2 and attack the ACE2-bearing cells lining the airways. It can infect upper as well as lower respiratory tracts and with the dying cells sloughing down and filling the airways the virus is carried deeper into the lungs. The thin layer of surfactant coating the airways becomes even thinner and the brush border less efficient to evict viruses and other foreign particles with a colder temperature and dry air, which may also dampen the immune response to the invading viruses. It appears that the virus is able to transmit while still confined to the upper airways, before invading the lower respiratory tract and lungs and causing severe symptoms. In addition, the virus is able to infect ACE2-bearing cells in other organs, including the blood vessels, gut and kidneys.

With the viral infestation, the activated immune system leads to macrophages to be recruited to the alveolar space, which increase cytokine production and attract additional immune cells such as T-helper cells CD4 and CD8 to the affected areas to combat the virus. The pattern recognition receptors (PRRs) of the immune cells recognize the virus and signal 
release of the pro-inflammatory cytokines such as interferon gamma (IFN-g), tumor necrosis factors (TNFs), interleukins (ILs), and chemokines. IFN-g activates macrophages which produce IL-6, TNF- $\alpha$, and IL-10. There are triggered additional pathways associated with PRRs, including cyclooxygenase (COX)-2 and c-Jun N-terminal kinase (JNK) with recovery, once the virus is cleared, the immune pathways shut down. However, the process goes into overdrive in a cytokine storm, initiating vascular leakage, coagulation cascades, and disseminated intravascular coagulation (DIC). Further, it leads to an increased susceptibility to infectious bacteria. Furthermore, the process affects other organs besides the lungs, especially in those suffering cronic diseases, leading to multi-organ failure (MOF).

Age is an important epidemiological factor. The elderly people are at risk of severe infections possibly because the ineffective initial anti-viral immune response. It appears that children may be less severely affected because their immune system is unlikely to progress to a cytokine storm. Further, there are other factors like individual genetic make-up, frequency of exposure, the amount of virus load, the other microbes in the body including gut microbiota which may play a role in acquirement of the infection and its progression. Directed by the viral RNA, the synthesis of polyprotein $1 \mathrm{a} / 1 \mathrm{ab}(\mathrm{pp} 1 \mathrm{a} / \mathrm{pp} 1 \mathrm{ab})$ in the host cells is brought about with the transcription occurring through the replication-transcription (RT) complex organized in doublemembrane vesicles (Figure 4).

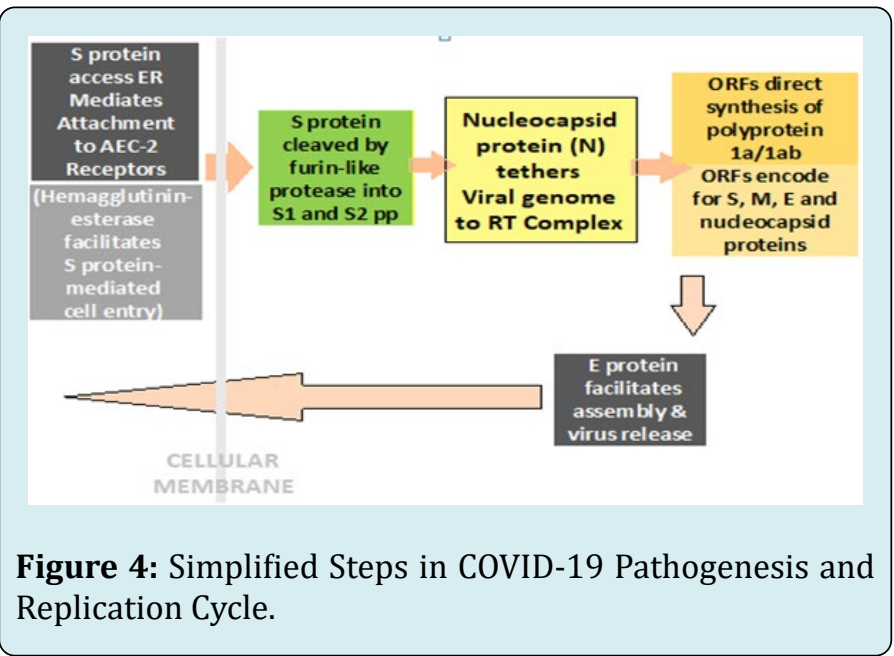

The open reading frames (ORFs) guide the production of both pp1a and pp1ab polypeptides which are processed by virally encoded chymotrypsin-like protease (3CLpro) or main protease (Mpro), as well as one or more papain-like proteases for producing 16 non-structural proteins (nsps). The ORFs also encode for structural proteins, including spike, membrane, envelope and nucleocapsid proteins. The pathophysiology and virulence mechanisms of COVID-19 virus are related to the function of the nsps and structural proteins. Further, the nsps appear to block the host innate immune response, as well. The E protein also plays a crucial role in promoting viral assembly and release of nascent virions.

\section{The Role of ACE2 Receptors in COVID-19}

It appears that the viral spike (S) protein plays an important role in viral attachment, fusion, and entry. ACE2 being the main host cell receptors for, plays a crucial role in the invasion of virus into the cell through the receptor binding domain (RBD) and infectivity and affliction of upper respiratory tract, lower respiratory tract and lungs, and gastrointestinal system apart from kidneys and various other organs. Considering this, the expression and distribution of the ACE2 in human body may outline the potential infection routes and the organs with high ACE2-expressing tissues are potential sites for 2019-nCoV infection. Further, the ACE2 expression is reduced in SARS-CoV infection and has been linked with the disease manifestations. In mice experiments, the injection of SARS-CoV Spike worsens acute lung failure in vivo, which can be attenuated by blocking the reninangiotensin pathway [7].

Significantly high ACE2 expression is found in type II alveolar cells (AT2) of lung, upper oesophageal stratified epithelial cells, absorptive enterocytes from ileum and colon, gall bladder and bile ductal cells, myocardial cells, kidney proximal tubule cells and bladder urothelial cells. In the lungs, the bulk of the ACE2-expressing cells, over 80 percent, are AT2 (alveolar type 2) cells. The abundant expression of ACE2 in the AT2 cells may explain the preferential involvement of lungs and severe alveolar damage following the infection. The distribution of ACE2 is more widespread in males than females, which is consistent with the epidemiological finding that men are more prone to COVID-19 than women. The ACE2 expressing of ACE2 AT2 cells also expresses many other genes favoring the viral processes. The ACE2 is also expressed on the oral mucosa and highly enriched in epithelial cells of tongue accounting for a potentially high risk of this route for 2019-nCoV infectious susceptibility. These findings may underline the prophylactic role of frequent mouth washing and rinsing [8]. Further, the saliva, urine and stool specimens and rectal swabs have demonstrated embedded viruses in the COVID-19 patients.

\section{The Multiple Strains of SARS-CoV-2}

The SARS-CoV-2 virus is mutating due to factors related to a neutral drift and random genetic changes. Initially, the virus which emerged from Wuhan was the D-strain. Currently, there are six strains of coronavirus. The original one is the $\mathrm{D}$ 
or L strain that appeared in Wuhan in December 2019. Its first mutation -- the S strain -- appeared at the beginning of 2020, while, since mid-January 2020, we have had strains $\mathrm{V}$ and $\mathrm{G}$. To date strain $\mathrm{G}$ is the most widespread and mutated into strains GR and GH at the end of February 2020. In North America, the most widespread strain is $\mathrm{GH}$, while in South America, it is the GR strain. Globally and in Asia the strains G, GH and GR are progressively increasing and by far the most widespread, representing about three-fourth of all [9]. They present four mutations, having changed sequence of the RNA polymerase and Spike proteins of the virus. The strain $S$ is found in some restricted areas in the US and Spain and the L and $V$ strains are gradually disappearing.

As now, in total, there have been identified over 285 mutations in SARS-CoV-2. But as such the mutations do not seem to have any impact on the disease severity. Though, the new G-strain, which is increasingly affecting populations of various countries, seems to be more contagious [10]. The new G-strain evolved as the result of a mutation known as D614G, has gradually replaced the $\mathrm{D}$ strain all over the world and now accounts for over $85 \%$ of published SARS-CoV-2 genomes [11]. In the new G-strain there is supplementation of aspartic acid (D) in the 614th position of the amino acid with glycine (G), hence, named as D614G. The D614G mutation which has occurred in the spike protein of the virus helps the virus in attaching more efficiently with the ACE2 receptor in the human host and thus appears to have increased infectivity. This mutation was first identified in China and afterwards in Europe, has spread to various countries including the U.S., Canada, and India.

Long et al sequenced the genomes of 5,085 SARS-CoV-2 strains causing COVID-19 waves in an ethnically diverse population of Houston in the USA. They concluded that most of the strains in the second wave were having a Gly614 amino acid replacement in the spike protein, which could be linked to increased transmission and infectivity [11]. The findings also help in delineating the origin and trajectory of subsequent infection waves.

The second and subsequent waves of the pandemic are also characterized by SARS-CoV-2 strains with diverse genotypes. Virtually all cases in the second and ongoing disease wave had been caused by strains with the Gly614 variant of spike protein. In the Gly614 variant, amino acid residue Asp614 is located at subdomain 2 (SD-2) of the spike protein and forms a hydrogen bond and electrostatic interaction with two adjacent residues in the S2 subunit. The replacement of aspartate with glycine possibly substantively weakens the bond between the S1 and S2 subunits, producing a highly fusogenic spike protein increasing the ability of S1 to dissociate from S2 and thus enhancing the Gly614 variant's ability to invade and enter the host cells. These findings highlight the importance of multiple importation events in the genetically diverse strains in epidemiology and pathogenesis of COVID-19.

\section{Fear, Confusion and Impact on Mass Behavior}

\section{The COVID-19 Situation, Distress and Behavioural Effect}

In most countries, fear levels were stated to rise in response to the mounting case count and governmental efforts to contain the pandemic through lockdown measures, over the last few months. The lockdown measures in general, have generated fears about the availability of essential goods and access to various amenities. The survey also found that while approving of the government's lockdown strategy, a significant number of citizens among various nations including Indians were fearful of the impact of lockdown on availability and quality of the essential services.

The spread of COVID-19 pandemic is a major public health concern threatening general and mental health and safety of the human life all over the globe [12]. There are a number of studies which have for assessed and validated of the anxiety and other psychiatric symptoms related to COVID-19 [13]. Another study involving general population, found that the anxiety levels and emotional stress were high, in general [14].

\section{Reaction to COVID-19 among Healthcare Professionals}

Assessment of the change in behavior and attitudes focusing on the healthcare providers' concern about getting infected and other related stress factors in context of the Covid-19 pandemic have been assessed in various studies. In general, there is a large concern about the aerosolized spread of the virus through being exposed to coughing respiratory patients and aerosol-generating procedures, associated with the apprehension that they may not be able to maintain a safe social distance appropriately. In addition, the anxiety and stress may also be caused by organizational factors and fear about resource shortages such as personal protective equipment (PPE) and protective masks are common, and concerns about not being able to provide competent care if deployed to a new area, about rapidly changing information and lack of communication, lack of specific drugs, the shortage of ventilators and intensive care unit beds necessary to take care for the surge of critically ill patients, and significant change in their own daily social and family life [15].

The healthcare professionals suffer with various concerns, which are different from the public at large. 
The responses from 8 listening sessions with groups of physicians, nurses, advanced practice clinicians, residents, and fellows have disclosed the sources of anxiety and concerns among healthcare workers. Hear me, protect me, prepare me, support me and care for me was the message from health care professionals dealing with the COVID-19 pandemic delivered to their organizations, according to an article published in JAMA [16]. On the part of the policy planners, along with maintaining critical supplies, there should be maintenance of an adequate healthcare workforce to maximize the ability of each healthcare worker to handle increased number patients and overall workload. The simple and genuine expressions of gratitude for the commitment of health care professionals should be properly supplemented by concrete support and facilities.

In general, the healthcare professionals are worried about their safety at the workplace and taking the infection home to their family, uncertainty that their organization will support for their personal and family needs if they develop infection, and issues about strenuous duties and long working hours and being able to provide competent medical care and access to up-to-date information and communication [17].

\section{Age Groups and the Perceived Threat to COVID-19}

Generations or the age-groups are often considered by their periodic span along the timeline, though there is no agreed upon formula to define the length of the span. In general, those born between 1981 to 2000 can be called Millennials (witnessed the dawn of the Millennium) or Generation Y. The Generation X is born between 1961 to 1980, whereas the Baby Boomers, Generation 0, are those born between 1941 to 1960 . Those born between 1921 to 1940 form the Silent or Generation Alpha. Whereas Generation Z (or Gen Z for short, or Zoomers) is the demographic cohort succeeding Millennials or Gen Y. Most members of Generation $\mathrm{Z}$ are born in the mid-to-late 1990s and the early 2010s, and most of the Gen $\mathrm{Z}$ have used digital technology since relatively young age and are comfortable with the Internet and social media, and are the successors of Gen Y [18].

Through the collected data, in the survey by YouGov and the Institute of Global Health Innovation, it has been inferred that among the populations groups within India and elsewhere, it is millennials or Gen Y who appear to be most fearful to COVID-19 infection compared to other age-groups. Among the millennials in India, women were more scared of the infection than men [19].

Across Generations, those belonging to the Gen $\mathrm{Z}$ generation (post-millennial adults) were less scared than millennials. Even the older generations (Gen X and older,
Gen Alpha), who are more vulnerable to covid-19 than millennials, were less afraid of the virus and the disease than millennials (Figure 5).

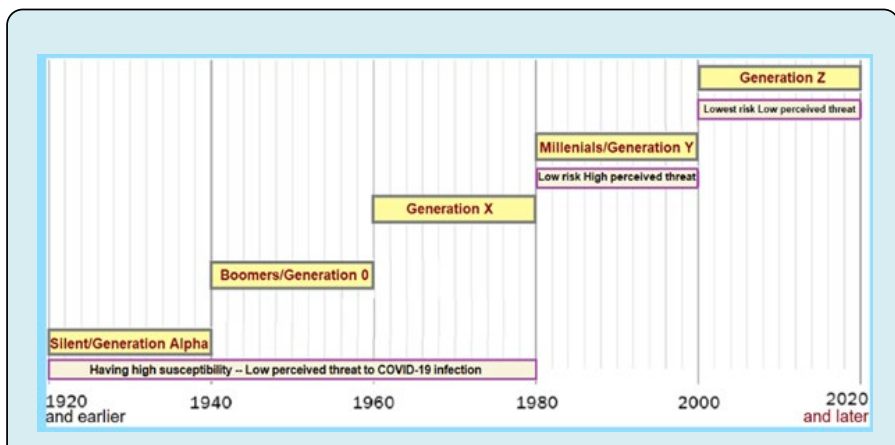

Figure 5: Age-groups and Generations Alpha, O, X, Y and Z and Risk and Perceived threat to COVID-19.

\section{Rational behavior for COVID-19 Prophylaxis}

As regards the fear to contracting the virus, respondents in Asian countries have reported taking more precautions (such as avoiding public places and wearing face masks) compared to Western peers. On most parameters, Indians ranked close to the median. The survey also noted that in case of personal hygiene (washing hands, using sanitizers, etc.), people from some countries were relatively less cautious about hygiene compared to others from more developed countries. The residents from most of the counties rated their governments' actions to contain the pandemic favorably. As per the survey, more than 80 percent respondents in Vietnam, India, and Denmark approved their government's response to handling COVID-19.

There are various surveys in offing and underway. The COVID-19 Symptoms \& Social Distancing Web Survey by Harvard aims to gather information on the prevalence of COVID-19 symptoms and social distancing behavior. Another survey, the Knowledge, Attitude and Practice Survey on COVID-19 is a platform to receive feedback from the public on measures to improve efforts in the fight against COVID-19 and encourage behavior change. There is also an International Survey on Coronaviruscovid19-survey.org to measure Worldwide COVID-19 Attitudes and Beliefs.

\section{Conclusion: The Lessons for Pandemic Control}

The human subjects produce respiratory droplets ranging from 0.1 to $1000 \mu \mathrm{m}$. Depending on droplet size, inertia, gravity, and evaporation factors, the emitted droplets and aerosols disperse in air. The large respiratory droplets will undergo gravitational settling faster than the smaller droplets and aerosols, which are likely to be buoyant and 
transported by air currents. The COVID-19 infection occurs through the transmission of virus-containing droplets $(>5$ to $10 \mu \mathrm{m})$ and aerosols $(\leq 5 \mu \mathrm{m})$ exhaled from infected individuals during breathing, speaking, coughing, and sneezing [20].

The aerosols can accumulate; remain infectious in indoor air for variable period depending on various environmental conditions. Thus, there are two obvious transmission pathways: the airborne inhalation and contracting through the contaminated surfaces. As appears, the contaminated surfaces may play less significant role in the disease transmission compared to the infected airborne droplets. Thus, the aerosol transmission is the major factor in the spread of COVID-19 infection [21]. Further, aerosols can remain airborne for hours, accumulate over time, and follow airflows over distances more than 6 feet [22]. The SARS-CoV-2 virions may be contained in submicron aerosols and can remain airborne for prolonged periods. Further, in a closed space with asymptomatic individuals, infectious aerosol concentrations may increase with the proportion of the time spent. The amount of ventilation, number of people present, the time spent in an indoor facility, and activities that affect airflow will influence the viral transmission pathways [23].

As there is a minimum virus titre required to cause COVID-19 infection, the viral load emitted in droplets; viability of the virus indoors and outdoors; mechanisms of transmission; concentrations in aerosols; and spatial patterns are important factors. There are uncertainties about SARS-CoV-2 transmission. The relative risk of transmission in various community settings is still unclear, as is the impact of mitigation measures in these contexts. Further, the effect of seasonality and heterogeneities in the population and immunity following the infection is not clear. The masks can protect uninfected individuals from SARS-CoV-2 aerosols and droplets. It is particularly important to wear masks in locations which are likely to accumulate high concentrations of the virus, such as health care settings, airplanes, restaurants, and other crowded places with reduced ventilation. The universal masking and social distancing are two most important sustainable options.

\section{References}

1. Meyerowitz EA, Richterman A, Gandhi RT, Sax PE (2020) Transmission of SARS-CoV-2: A Review of Viral, Host, and Environmental Factors. Ann Intern Med.

2. Byrne AW, McEvoy D, Collins AB, Hunt K, Casey M, et al. (2020) Inferred duration of infectious period of SARSCoV-2: rapid scoping review and analysis of available evidence for asymptomatic and symptomatic COVID-19 cases. BMJ Open 10(8): e039856.

3. Laxminarayan R, Wahl B, Dudala SR, Gopal K, Chandra $\mathrm{MB}$, et al. (2020) Epidemiology and transmission dynamics of COVID-19 in two Indian states. Science 370(6517): 691-697.

4. Vlachakis D, Papakonstantinou E, Mitsis T, Pierouli K, Diakou I, et al. (2020) Molecular mechanisms of the novel coronavirus SARS-CoV-2 and potential anti-COVID19 pharmacological targets since the outbreak of the pandemic. Food and Chemical Toxicology 146(111805).

5. Scheuch G (2020) Breathing Is Enough: For the Spread of Influenza Virus and SARS-CoV-2 by Breathing Only. J Aerosol Med Pulm Drug Deliv 33(4): 230-234.

6. Lee EC, Wada NI, Grabowski MK, Gurley ES, Lessler J (2020) The engines of SARS-CoV-2 spread. Science 370(6515): 406-407.

7. Imai Y, Kuba K, Penninger JM (2008) The discovery of angiotensin-converting enzyme 2 and its role in acute lung injury in mice. Exp Physiol 93(5): 543-548.

8. Nikhra V (2020) Exploring pathophysiology of COVID-19 infection: faux espoir and dormant therapeutic options. Int J Clin Virol 4: 34-40.

9. Mercatelli D, Giorgi FM (2020) Geographic and Genomic Distribution of SARS-CoV-2 Mutations. Frontiers in Microbiology.

10. Korber B, Fischer WM, Gnanakaran S, Yoon H, Theiler J, et al. (2020) Tracking Changes in SARS-CoV-2 Spike: Evidence that D614G Increases Infectivity of the COVID-19 Virus. Cell 182(4): 812-847.

11. Long SW, Olsen RJ, Christensen PA, Bernard DW, Davis JJ, et al. (2020) Molecular Architecture of Early Dissemination and Massive Second Wave of the SARSCoV-2 Virus in a Major Metropolitan Area. mBIO.

12. Ahorsu DK, Lin CY, Imani V, Saffari M, Griffiths MD, et al. (2020) The Fear of COVID-19 Scale: Development and Initial Validation. Int J Ment Health Addict, pp: 1-9.

13. Soraci P, Ferrari A, Abbiati FA, Fante ED, Pace RD, et al. (2020) Validation and Psychometric Evaluation of the Italian Version of the Fear of COVID-19 Scale. Int J Ment Health Addict 1-10.

14. Ugbolue UC, Duclos M, Urzeala C, Berthon M, Kulik k, et al. (2020) An Assessment of the Novel COVISTRESS Questionnaire: COVID-19 Impact on Physical Activity, Sedentary Action and Psychological Emotion. J Clin Med 9(10): 3352. 
15. El-Hage $W$, Hingray C, Lemogne C, Yrondi A, Brunault $P$, et al. (2020) Health Professionals Facing the Coronavirus Disease 2019 (COVID-19) Pandemic: What Are the Mental Health Risks?. Encephale 73-80.

16. Shanafelt T, Ripp J, Trockel M (2020) Understanding and addressing sources of anxiety among health care professionals during the COVID-19 pandemic. JAMA 323(21): 2133-2134.

17. Weilenmann S, Ernst J, Petry H, Sazpinar O, Pfaltz MC, et al. (2020) Health Care Workers' Mental Health During the First Weeks of the SARS-CoV-2 Pandemic in Switzerland: A Cross-Sectional Study. MedRxiv preprint.

18. Wang C, Pan R, Wan X, Tan Y, Xu L, et al. (2020) Immediate Psychological Responses and Associated Factors during the Initial Stage of the 2019 Coronavirus Disease (COVID-19) Epidemic among the General Population in China. Int J Environ Res Public Health 17(5): 1729.
19. Roy D, Tripathy S, Kar SK, Sharma N, Verma SK, et al. (2020) Study of knowledge, attitude, anxiety \& perceived mental healthcare need in Indian population during COVID-19 pandemic. Asian J Psychiatr 51: 102083.

20. Prather KA, Wang CC, Schooley RT (2020) Reducing transmission of SARS-CoV-2. Science 368(6498): 14221424.

21. Netz RR, Eaton WA (2020) Physics of virus transmission by speaking droplets. PNAS 117(41): 25209-25211.

22. Morawska L, Cao J (2020) Airborne transmission of SARS-CoV-2: The world should face the reality. Environ Int 139: 105730.

23. Buonanno G, Stabile L, Morawska L (2020) Estimation of airborne viral emission: Quanta emission rate of SARSCoV-2 for infection risk assessment. Environ Int 141: 105794. 